\title{
Pulse pair beamforming and the effects of reflectivity field variations on imaging radars
}

\author{
Boon Leng Cheong, Michael W. Hoffman, and Robert D. Palmer \\ Department of Electrical Engineering, University of Nebraska, Lincoln, Nebraska, USA
}

Stephen J. Frasier and F. J. López-Dekker

Department of Electrical and Computer Engineering, University of Massachusetts, Amherst, Massachusetts, USA

Received 22 November 2002; revised 3 May 2004; accepted 6 May 2004; published 16 June 2004.

[1] Coherent radar imaging (CRI), which is fundamentally a beamforming process, has been used to create images of microscale, reflectivity structures within the resolution volume of atmospheric Doppler radars. This powerful technique has the potential to unlock many new discoveries in atmospheric studies. The Turbulent Eddy Profiler (TEP) is a unique $915 \mathrm{MHz}$ boundary layer radar consisting of a maximum of 91 independent receivers. The TEP configuration allows sophisticated CRI algorithms to be implemented providing significant improvement in angular resolution. The present work includes a thorough simulation study of some of the capabilities of the TEP system. The pulse pair processor, used for radial velocity and spectral width estimation with meteorological radars, is combined with beamforming technique, in an efficient manner, to the imaging radar case. By numerical simulation the new technique is shown to provide robust and computationally efficient estimates of the spectral moments. For this study, a recently developed atmospheric radar simulation method is employed that uses the ten thousand scattering points necessary for the high resolution imaging simulation. Previous methods were limited in the number of scatterers due to complexity issues. Radial velocity images from the beamforming radar are used to estimate the three-dimensional wind field map within the resolution volume. It is shown that a large root mean square (RMS) error in imputed three-dimensional wind fields can occur using standard Fourier imaging. This RMS error does not improve even as SNR is increased. The cause of the error is reflectivity variations within the resolution volume. The finite beamwidth of the beamformer skews the radial velocity estimate, and this results in poor wind field estimates. Adaptive Capon beamforming consistently outperforms the Fourier method in the quantitative study and has been demonstrated to enhance the performance compared to the Fourier method. INDEX TERMS: 6969 Radio Science: Remote sensing; 6974 Radio Science: Signal processing; 6982 Radio Science: Tomography and imaging; 6994 Radio Science: Instruments and techniques; KEYWORDS: pulse pair beamforming, gradient variations, imaging radars

Citation: Cheong, B. L., M. W. Hoffman, R. D. Palmer, S. J. Frasier, and F. J. López-Dekker (2004), Pulse pair beamforming and the effects of reflectivity field variations on imaging radars, Radio Sci., 39, RS3014, doi:10.1029/2002RS002843.

\section{Introduction}

[2] Imaging radars have recently become an important tool for high-resolution remote sensing of the atmospheric boundary layer (ABL) [Mead et al.,

Copyright 2004 by the American Geophysical Union. 0048-6604/04/2002RS002843\$11.00
1998; Pollard et al., 2000], the mesosphere [Yu et al., 2001; Hysell et al., 2002], and the thermosphere [Hysell, 1996; Hysell and Woodman, 1997]. Through the use of coherent radar imaging (CRI), as it is termed in the atmospheric radar community, observations of small-scale structure in the reflectivity and wind fields are possible. CRI can be applied to radar systems with numerous, spatially separated, independent receivers. Much of the progress on CRI stems from the pioneering 
work in spatial interferometry (SI) [Pfister, 1971; Woodman, 1971]. Since that time, many interesting studies have taken advantage of the improved angular resolution offered by SI [e.g., Röttger and Vincent, 1978; Röttger and Ierkic, 1985; Kudeki and Woodman, 1990; Palmer et al., 1995].

[3] Techniques similar to CRI have been used for a wide range of applications, from seismology, sonar, medical imaging, and radio astronomy [e.g., Haykin, 1985]. The term beamforming is used in many other fields to describe the imaging process. The first use of CRI for probing of the atmosphere was provided by Kudeki and Sürücü [1991], who used the Jicamarca Radio Observatory for observations of the equatorial electrojet. They used a Fourier-based method for imaging which has well-known resolution limitations. In an effort to increase angular resolution, the Maximum Entropy method, made popular in the radio astronomy community, has been used for imaging of $F$ region irregularities [Hysell, 1996; Hysell and Woodman, 1997]. Subsequently, much research has been conducted related to finding the optimum algorithm, in terms of angular resolution, computational complexity, and robustness [Yu et al., 2000]. For example, the Capon algorithm [Capon, 1969] has proven to have moderate computational complexity and resolution approaching that of the Maximum Entropy method under certain conditions [Palmer et al., 1998].

[4] A generalized presentation of CRI by Woodman [1997] provided a mathematical foundation for this promisingtechnique. Further, many statistical studies have been presented in the literature comparing the various algorithms available for CRI [e.g., Yu et al., 2000; Chau and Woodman, 2001]. Undoubtedly, new algorithms will be devised with ever improving resolution and robustness.

[5] Given these technical and scientific advances, the use of CRI is becoming commonplace in newly developed atmospheric radar systems. For example, a French stratosphere-troposphere (ST) radar has recently been outfitted with CRI capabilities allowing intriguing studies of aspect sensitivity at VHF wavelengths [Hélal et al., 2001]. New commercial systems are also being deployed throughout the world with multiple receivers capable of CRI research [e.g., Yu et al., 2001]. In addition to the Jicamarca Radio Observatory, many of the most powerful mesosphere-stratosphere-troposphere (MST) radars are being upgraded to allow CRI studies. For example, the MU radar [Fukao et al., 1985a, 1985b], which was originally designed with only four independent receivers, is currently being enhanced to 25 receivers. This exciting modification will provide significant CRI capabilities. As another example, researchers at the Indian MST radar facility [Rao et al., 1995; Jain et al., 1995] are designing independent receive arrays with the intent of implementing CRI. For a thorough review of the advantages of CRI, and other related techniques, the reader is referred to an article by Luce et al. [2001]. With these recent developments and previous research accomplishments, it is apparent that CRI is becoming an important tool for studies of the atmosphere.

[6] As mentioned earlier, the work reviewed has found application throughout all atmospheric layers. Typically, the radar systems used for this research were limited to several independent receivers. For the purpose of imaging the $\mathrm{ABL}$, a $915 \mathrm{MHz}$ imaging radar was developed by researchers at the University of Massachusetts-Amherst [Mead et al., 1998; Pollard et al., 2000], and was called the Turbulent Eddy Profiler (TEP). This system is capable of observing the ABL in a $25^{\circ}$ cone above the surface using 61-91 independent receiving antennas, depending on the configuration. This unique design allows the use of sophisticated beamforming/imaging algorithms, which have numerous advantages.

[7] The present study details recent advances that are applicable to imaging radars for high-resolution measurements of the reflectivity and wind fields. Many of the results are based on numerical simulations of the TEP radar configuration. Although this specific system is simulated, the results are generally applicable. In section 2 an efficient algorithm is presented for spectral moment estimation using imaging radars. A new numerical simulation method recently developed by Cheong et al. [2004] was used in order to properly and efficiently emulate the high angular resolution TEP system. These results will be provided in section 3 providing wind field estimates for a variety of reflectivity fields. Finally, conclusions are provided in the final section.

\section{Pulse Pair Beamforming}

[8] CRI, or beamforming, provides an estimate of the received signal from distinct angular positions within the transmit beam of the radar. This is accomplished by coherently combining signals arriving at different spatial locations in order to estimate the signal for a particular direction. Often, the signal is used to estimate the echo power but there is no restriction that only this parameter can be obtained. Given a radar array positioned on the ground, it is possible to image within a particular solid angle for each range. One can create an image by raster scanning through a desired $x$ and $y$ range. If echo power is calculated from the signal, the resulting map, or image, is proportional to the reflectivity within the beam of the radar. Using a similar notation to that of Palmer et al. [1998], let $\mathbf{x}(t)$ represent a vector of received signals from $n$ sensors. 
By combining these $n$ signals with the complex coefficients in $\mathbf{w}$, the output of the beamformer at time $t$ will be given by complex scalar $y(t)$ as

$$
y(t)=\mathbf{w}^{\dagger} \mathbf{x}(t)
$$

where the dagger represents the Hermitian operator (conjugate transpose). Typically, the elements of $\mathbf{w}$ are chosen to create constructive interference at a particular angle. The resulting signal could be used directly to calculate the Doppler spectrum. However, this computational burden is not needed if the three spectral moments would suffice.

[9] The autocovariance function of the output signal can be expressed as

$$
\begin{aligned}
R_{y y}(\tau) & =E\left\{y(t) y^{\dagger}(t-\tau)\right\} \\
& =E\left\{\mathbf{w}^{\dagger} \mathbf{x}(t) \mathbf{x}^{\dagger}(t-\tau) \mathbf{w}\right\} \\
& =\mathbf{w}^{\dagger} \mathbf{R}_{\mathbf{x x}}(\tau) \mathbf{w}
\end{aligned}
$$

where $\mathbf{R}_{\mathbf{x} \mathbf{x}}(\tau)$ is the autocovariance matrix of the received signals in $\mathbf{x}(t)$.

$$
\mathbf{R}_{\mathbf{x x}}(\tau)=\left[\begin{array}{cccc}
R_{11}(\tau) & R_{12}(\tau) & \ldots & R_{1 n}(\tau) \\
R_{21}(\tau) & R_{22}(\tau) & \ldots & R_{2 n}(\tau) \\
\vdots & \vdots & \ddots & \vdots \\
R_{n 1}(\tau) & R_{n 2}(\tau) & \ldots & R_{n n}(\tau)
\end{array}\right]
$$

Each element in $\mathbf{R}_{\mathbf{x x}}(\tau)$ represents the autocovariance of signals from a pair of receivers.

[10] A typical choice of $\mathbf{w}$, which provides unity gain in the direction of the wavenumber vector $\mathbf{k}$, is called the Fourier imaging method and the weight vector is given by

$$
\mathbf{w}_{f}=\frac{1}{\sqrt{n}}\left[e^{j \mathbf{k} \cdot \mathbf{D}_{1}} e^{j \mathbf{k} \cdot \mathbf{D}_{2}} \ldots e^{j \mathbf{k} \cdot \mathbf{D}_{\mathbf{n}}}\right]^{T}
$$

where $\mathbf{k}=\frac{2 \pi}{\lambda}[\sin \theta \sin \phi \sin \theta \cos \phi \cos \theta]$, with $\theta$ and $\phi$ as the zenith and azimuth angles, respectively. $\mathbf{D}_{i}$ represents the position vector for the phase center of receiver $i$. By substituting $\mathbf{w}_{f}$ into equation (1), we obtain the Fourier-based estimate of the output sequence.

[11] Using the concept behind the pulse pair processor [Doviak and Zrnić, 1993], the power, radial velocity and spectral width for direction $\mathbf{k}$ can be calculated using a temporal sampling of $R_{y y}(\tau)$ at $\tau=T_{s}$. The parameters can be expressed as follows:

$$
\begin{array}{r}
P(\mathbf{k})=R_{y y}(0)=\mathbf{w}^{\dagger} \mathbf{R}_{\mathbf{x x}}(0) \mathbf{w} \\
v_{r}(\mathbf{k})=-\frac{\lambda}{4 \pi T_{s}} \arg \left[R_{y y}\left(T_{s}\right)\right] \\
=-\frac{\lambda}{4 \pi T_{s}} \arg \left[\mathbf{w}^{\dagger} \mathbf{R}_{\mathbf{x x}}\left(T_{s}\right) \mathbf{w}\right]
\end{array}
$$

$$
\begin{aligned}
\sigma_{v}(\mathbf{k}) & =\frac{\lambda}{2 \pi T_{s} \sqrt{2}}\left|\ln \left(\frac{S}{\left|R_{y y}\left(T_{S}\right)\right|}\right)\right|^{1 / 2} \\
& =\frac{\lambda}{2 \pi T_{s} \sqrt{2}}\left|\ln \left(\frac{S}{\left|\mathbf{w}^{\dagger} \mathbf{R}_{\mathbf{x x}}\left(T_{s}\right) \mathbf{w}\right|}\right)\right|^{1 / 2}
\end{aligned}
$$

where $S$ is the signal power. In practice, $\mathbf{R}_{\mathbf{x x}}(0), \mathbf{R}_{\mathbf{x x}}\left(T_{S}\right)$ and $S$ are replaced by their estimates. One could estimate the noise power from the "spike" of the autocorrelation function at lag-0 when no signal is present and subtract that from $R_{y y}(0)$ to obtain $\hat{S}$ the estimate of signal power $S$. The combined technique of pulse pair processing and beamforming is termed pulse pair beamforming (PPB). In essence, PPB exploits the linear relationship between the beamformer inputs and output to generate covariance lags of the output by using those that are computed for the inputs. The pulse pair relationship for the various spectral moments can be computed based on input correlations as opposed to the correlations of the outputs. This enhances our ability to image inside the beam of the radar. While computationally less efficient than conventional weight-and-sum beamforming, this formulation is advantageous in adaptive algorithms that require computation of the covariance matrix to determine the weights. Hence it provides an efficient method of estimating the spectral moments for imaging applications using adaptive algorithms. It should be noted that many of the concepts behind PPB can be related to the early work of Kudeki and Woodman [1990] and Palmer et al. [1993].

[12] With this obvious extension of the pulse pair processor to imaging applications, one can make use of the extensive literature on the statistical characteristics of the pulse pair processor, in general [see Doviak and Zrnić, 1993, and references therein]. It should be noted that the PPB algorithm described in the previous equations requires estimation of the covariance function. Standard estimation schemes, including incoherent integration, etc., can be employed for this purpose. Fourier imaging techniques are simple to implement and efficient algorithms such as FFT-based implementations can be used for various applications. However, it is well known that Fourier-based algorithms also suffer in the presence of clutter due to the fixed high sidelobes and have limited resolution. More sophisticated adaptive methods were developed in the late 1960 s, producing higher resolution and the ability to null the interfering signals coming from ground clutter, for example. One such technique is known as Capon's method [Capon, 1969] but is also referred to as the Minimum Variance Method. Since the Fourier beamformer has a fixed beam pattern, which behaves similar to $\sin (x) / x$, it inherently has fixed-gain sidelobes. Strong echoes can leak through sidelobes to the region of interest, possibly masking the desired 
signal. The Capon method attempts to minimize the output power of the beamformer with the constraint of maintaining unity gain in the desired direction. The minimization helps reduce leakage of high power signals through antenna pattern sidelobes. The optimization problem can be expressed mathematically as

$$
\min _{\mathbf{w}} P(\mathbf{k}) \quad \text { subject to } \mathbf{e}^{\dagger} \mathbf{w}=1
$$

where $\mathbf{e}=\left[e^{j \mathbf{k} \cdot \mathbf{D}_{1}} e^{j \mathbf{k} \cdot \mathbf{D}_{2}} \ldots e^{j \mathbf{k} \cdot \mathbf{D}_{\mathbf{n}}}\right]^{T}$ is the steering vector for the look direction resulting in wavenumber vector $\mathbf{k}$.

[13] Intuitively, Capon's method only maintains unity gain in the beamformer direction defined by $\mathbf{k}$. The algorithm has freedom in the choice of the gain for other directions. As a result, Capon's method may produce higher gain in directions where there is little backscatter energy. Of course, this higher gain has little adverse effect given that the backscattered energy is low.

[14] This constrained minimization problem can be solved using Lagrangian methods, resulting in the wellknown solution for the weight vector

$$
\mathbf{w}_{c}=\frac{\mathbf{R}_{\mathbf{x x}}{ }^{-1}(0) \mathbf{e}}{\mathbf{e}^{\dagger} \mathbf{R}_{\mathbf{x x}}{ }^{-1}(0) \mathbf{e}}
$$

In general, it was found that higher resolution is achieved in generating the output power map using Capon's method compared to the Fourier method [Palmer et al., 1998]. It should be emphasized that the Capon weight vector was derived based on the idea of minimizing output power. No attempt was made to mitigate any effects on the estimated radial velocity. It will be shown later that the reflectivity field does have a significant effect on the imaged wind field map.

\section{Simulation Results}

\subsection{Simulation Technique Overview}

[15] The simulation model developed by Holdsworth and Reid [1995] has proven to be invaluable for diverse studies of atmospheric radar applications [e.g., $Y u, 2000]$. However, the computational complexity of the required spatial filter becomes limiting for a large number of scatterers. This method was applied to a simulation of the TEP system at $915 \mathrm{MHz}$. Owing to the large number of receivers and the high resolving capacity of the TEP array, however, the limited number of scatterers proved to be ineffective in emulating volume scattering. Therefore the modified scheme proposed by Cheong et al. [2004] is used for the current study. This method is similar to that of Holdsworth and Reid [1995] but uses a table look-up method and linear interpolation. A set of pregenerated tables for a turbulent field are stored in a standardized manner that consists of velocity samples over a uniform three-dimensional grid within the enclos- ing volume. For each scatterer position, the turbulent velocity is extracted from the table and linearly interpolated among the eight surrounding points within a cubical region. As time evolves, the tabulated turbulent values are updated reflecting the time change behavior of the turbulent field.

[16] The tabulated values of the turbulent field can be generated in any number of ways. For example, the previous method of Holdsworth and Reid [1995] could be used to generate the sequence of turbulent look-up tables. Since the turbulent fields are not updated at each instance in time and only linear interpolation is used, the computational efficiency gain is tremendous. Other physical-based methods of generating the turbulent fields could be used without increasing the complexity of the method. A interesting example was provided by Muschinski et al. [1999], who used the resulting fields from a large eddy simulation to generated Doppler radar data.

\subsection{Turbulent Eddy Profiler Configuration}

[17] For this simulation, the TEP array is configured to have up to 61 sensors arranged in a hexagonal lattice. Each sensor is separated by approximately $0.5412 \mathrm{~m}$, which is based on the actual TEP system. The range resolution is set to $30 \mathrm{~m}$. A vertically directed transmit beam is used with a one-way, half-power beamwidth of $25^{\circ}$. Including coherent integration, the backscattered signal is sampled at a period of 0.007143 s. Figure 1 shows the simulated configuration of TEP array.

\subsection{Consistency of Pulse Pair Beamforming}

[18] Using the method of Cheong et al. [2004], time series data were generated for the simulated TEP array. The general parameters of the simulation are provided in Table 1.

[19] As a consistency check of the simulation method, Figure 2 provides several examples of Doppler spectra for five distinct pointing directions using both the Fourier and Capon beamformers. For this simple example, a symmetric, two-dimensional Gaussian reflectivity field with half-power width of $10^{\circ}$ was used. The top panels provide the simulated radial velocity field within $\pm 12.5^{\circ}$ of zenith. For each pointing direction, the expected first moment of the Doppler spectrum will change. As we can see, the Doppler spectra are consistent with the radial velocity map. Since the wind is flowing at $25 \mathrm{~ms}^{-1}$ with $45^{\circ}$ azimuth angle without vertical velocity $(u=-17.67$, $v=-17.67$ and $w=0 \mathrm{~ms}^{-1}$ ), one would expect negative radial velocity in the upper right region and positive radial velocity in the lower left region. Direction (1) is pointing vertically and shows the expected small radial velocity since the simulated vertical velocity is zero, in this case. The other four points also yield reasonable radial velocities and Doppler spectra. The actual values of radial velocity at each of these five points is indicated 


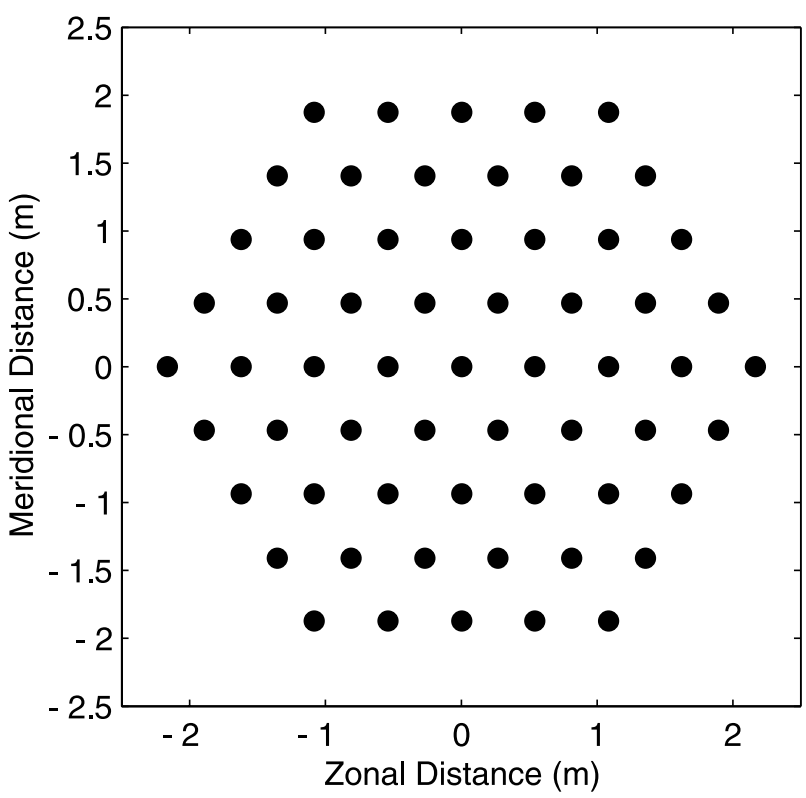

Figure 1. The TEP array is configured to have 61 sensors arranged in a hexagonal lattice. Each sensor is separated approximately $0.5412 \mathrm{~m}$.

in the figure next to the panels for each of the five points. Note that the $0.3 \mathrm{~ms}^{-1}$ RMS turbulent velocities result in small but non-zero vertical radial velocities.

\subsection{Simulated Experimental Setup}

[20] An example reflectivity model used in the simulation is shown in Figure 3. It is composed of two elongated Gaussian blobs, each with the following form:

$$
P(x, y)=\frac{1}{2 \pi \sigma_{x} \sigma_{y} \sqrt{1-\rho^{2}}} \exp \left[-\frac{B}{2\left(1-\rho^{2}\right)}\right]
$$

where

$$
B=\frac{\left(x-\mu_{x}\right)^{2}}{\sigma_{x}^{2}}+\frac{\left(y-\mu_{y}\right)^{2}}{\sigma_{y}^{2}}-\frac{2 \rho\left(x-\mu_{x}\right)\left(y-\mu_{y}\right)}{\sigma_{x} \sigma_{y}}
$$

Table 1. List of Simulation Parameters

\begin{tabular}{ll}
\hline \multicolumn{1}{c}{ Parameter } & \multicolumn{1}{c}{ Value } \\
\hline Range & $1000 \mathrm{~m}$ \\
Transmitter pointing direction & vertical \\
Sampling time, $T_{s}$ & $0.007143 \mathrm{~s}$ \\
Number of scatterers & 10,000 \\
Horizontal wind magnitude & $25 \mathrm{~ms}^{-1}$ \\
Azimuth angle & $45^{\circ}$ \\
Vertical wind & $0 \mathrm{~ms}^{-1}$ \\
Turbulent RMS velocity & $0.3 \mathrm{~ms}^{-1}$ \\
\hline
\end{tabular}
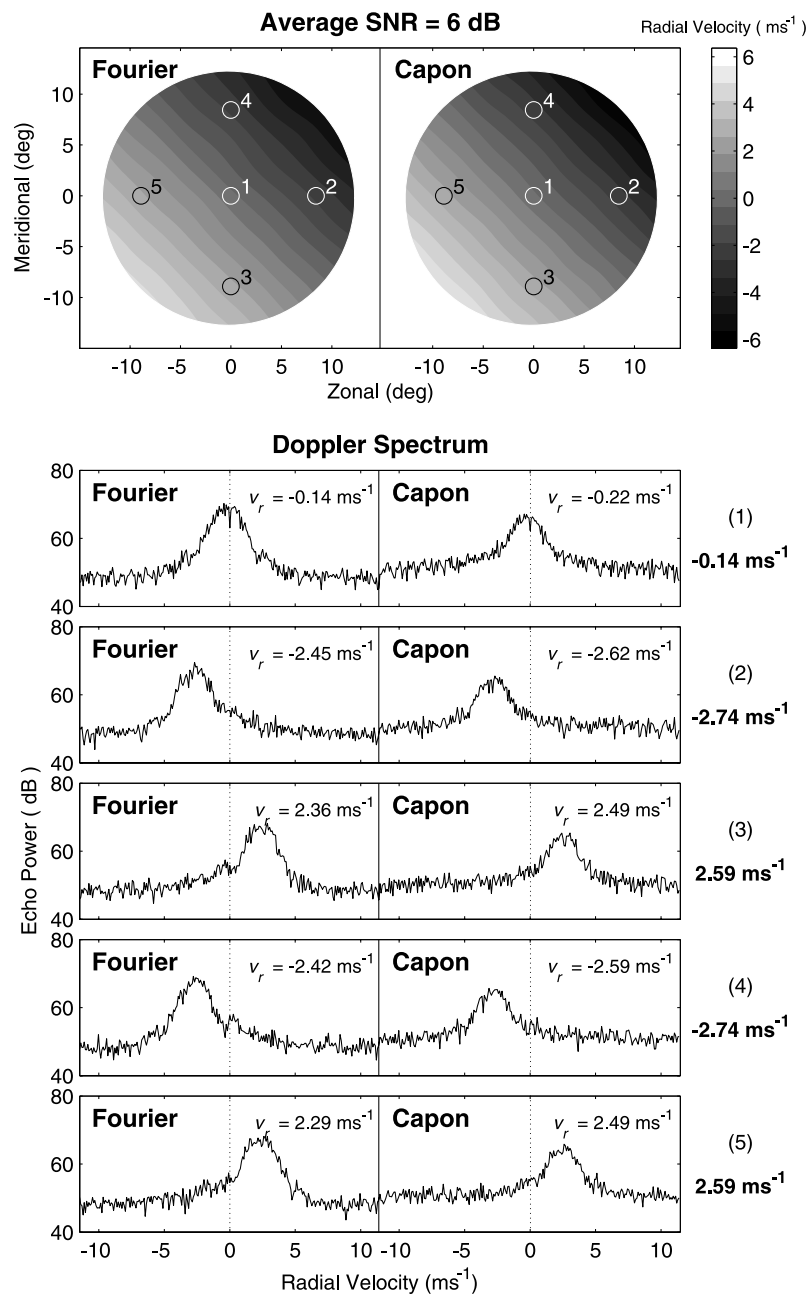

Figure 2. Example Doppler spectra from the simulation method. Horizontal wind has a speed of $25 \mathrm{~ms}^{-1}$ with $45^{\circ}$ azimuth angle with zero vertical velocity. Top panels show the images obtained using Fourier and Capon PPB methods. Radial velocity estimates for the chosen five pixels are listed at the upper right corner of the plot. The true velocity is stated to the right in bold.

and

$$
\rho=\frac{\sigma_{x y}}{\sigma_{x} \sigma_{y}}
$$

[21] The two scattering centers are located at $\left(2^{\circ}, 4^{\circ}\right)$ and $\left(-6^{\circ},-4^{\circ}\right)$ with $\sigma_{x}=2^{\circ}, \sigma_{y}=4^{\circ}, \rho=-0.75$, and $\sigma_{x}=2^{\circ}, \sigma_{y}=4^{\circ}, \rho=-0.5$, respectively. For this simulation a northeasterly wind is used with an azimuth angle of $45^{\circ}$ and mean velocity of $25 \mathrm{~ms}^{-1}$. Isotropic turbulence was introduced to the wind field model with an RMS value of $0.3 \mathrm{~ms}^{-1}$. A white noise sequence is 


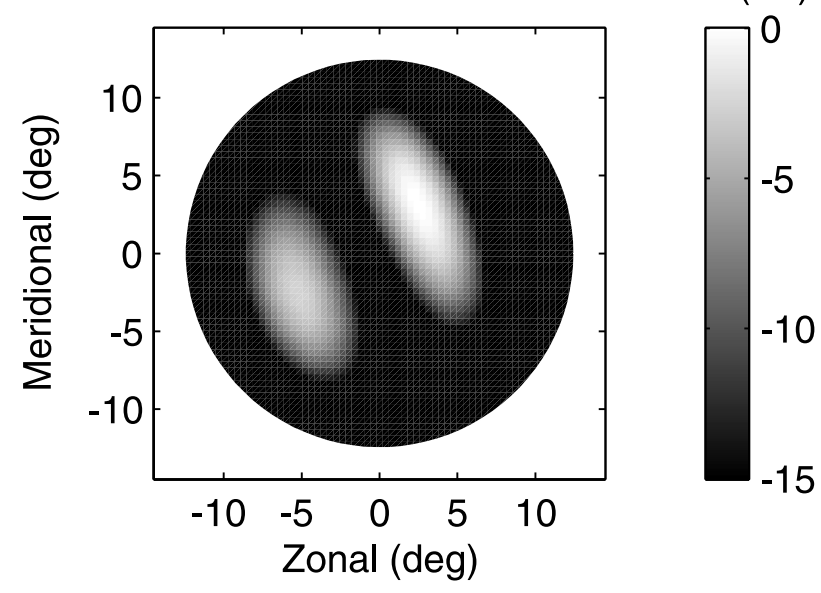

Figure 3. Reflectivity model used in the simulation comprised of two Gaussian-shaped blobs centered at $\left(2^{\circ}\right.$, $4^{\circ}$, and $\left(-6^{\circ},-4^{\circ}\right)$ with $\sigma_{x}=2^{\circ}, \sigma_{y}=4^{\circ}, \rho=-0.75$ and $\sigma_{x}=2^{\circ}, \sigma_{y}=4^{\circ}, \rho=-0.5$, respectively.

added to each receiver signal to give an SNR of $3 \mathrm{~dB}$ for each sensor. The new simulation method allowed the use of 10,000 scattering points and the results are presented in the following section.

\subsubsection{Moment Maps}

[22] In this section, results using the new PPB method are provided. In Figure 4 the top panel shows echo power obtained by CRI using both Fourier and Capon based methods.

[23] The middle panel shows the corresponding radial velocity estimate using the new PPB method. The weight vectors used in equation (6) are both obtained from the Fourier and Capon procedures. As expected, the radial velocity contour lines derived from the PPB method are generally perpendicular to the wind direction. The bottom panel shows the spectral width estimate using equation (7). As stated previously, these maps were obtained using 10,000 scatterers in the volume. Note that radial velocity and spectral width maps are shown for regions with SNR $>3 \mathrm{~dB}$.

[24] Given a wind magnitude of $25 \mathrm{~ms}^{-1}$, radial velocities of $-6.45 \mathrm{~ms}^{-1}$ and $+6.45 \mathrm{~ms}^{-1}$ should be observed at the edge of the circle $\left(12.5^{\circ}\right)$ at northeast and southwest, respectively. The estimates are shown in the middle panel of Figure 4 where visually accurate results are obtained. A more thorough statistical comparison will be provided when the radial velocity estimates are subsequently used to calculate the three-dimensional wind field.

[25] Spectral width estimates are shown in the bottom panel of Figure 4. The estimates have been normalized by $2 v_{a}$ where $v_{a}=11.47 \mathrm{~ms}^{-1}$ for this simulated TEP configuration. Although a direct comparison to simulated values is difficult due to beam broadening effects, the spectral width estimates using the Fourier method show consistent results in regions of high reflectivity. Since the RMS value of the simulated turbulence is constant across the map, an approximately constant spectral width estimate should be expected. In contrast, the spectral width estimates from the Capon method are erratic and not comparable to the Fourier results. This is due to the adaptive nature the Capon algorithm which changes the shape of the beam pattern for each pointing direction. Of course, the adaptation is a result of the constrained minimization problem stated in equation (8). As will be seen in the next section, the adaptive nature of the Capon algorithm has a positive effect on the estimation of the reflectivity and velocity fields. However, the spectral width estimates are profoundly affected by the beam broadening effect which will change for each pointing direction using the Capon method. Therefore it is recommended to use the Fourier or other fixed beamforming methods for spectral width estimates.

\subsubsection{Wind Field Maps}

[26] Similar to the standard Doppler Beam Swinging (DBS) method, an estimate of the three-dimensional wind field map can be obtained by solving the following set of linear equations for each pixel in the map [e.g., Pollard et al., 2000].

$$
\mathbf{C u}=\mathbf{v}_{r}
$$

where

$$
\begin{aligned}
& \mathbf{C}=\left[\begin{array}{ccc}
\sin \theta_{1} \sin \phi_{1} & \sin \theta_{1} \cos \phi_{1} & \cos \theta_{1} \\
\sin \theta_{2} \sin \phi_{2} & \sin \theta_{2} \cos \phi_{2} & \cos \theta_{2} \\
\sin \theta_{3} \sin \phi_{3} & \sin \theta_{3} \cos \phi_{3} & \cos \theta_{3} \\
\vdots & \vdots & \vdots \\
\sin \theta_{m} \sin \phi_{m} & \sin \theta_{m} \cos \phi_{m} & \cos \theta_{m}
\end{array}\right] \\
& \mathbf{u}=\left[\begin{array}{c}
u \\
v \\
w
\end{array}\right] \quad \mathbf{v}_{r}=\left[\begin{array}{c}
v_{r}^{(1)} \\
v_{r}^{(2)} \\
v_{r}^{(3)} \\
\vdots \\
v_{r}^{(m)}
\end{array}\right]
\end{aligned}
$$

where $\theta_{i}$ and $\phi_{i}$ are the azimuth and zenith angles for pixel $i$, respectively. For this study, a $3 \times 3$ sliding window is used providing nine equations for the estimate of the wind field. This sliding window may progress across the map in an overlapped or non-overlapped manner. Figure 5 illustrates the $3 \times 3$ sliding window for extracting 9 pixels used in computing the wind field for the middle pixel. As in the standard DBS method, it is assumed that the wind field is constant across the 

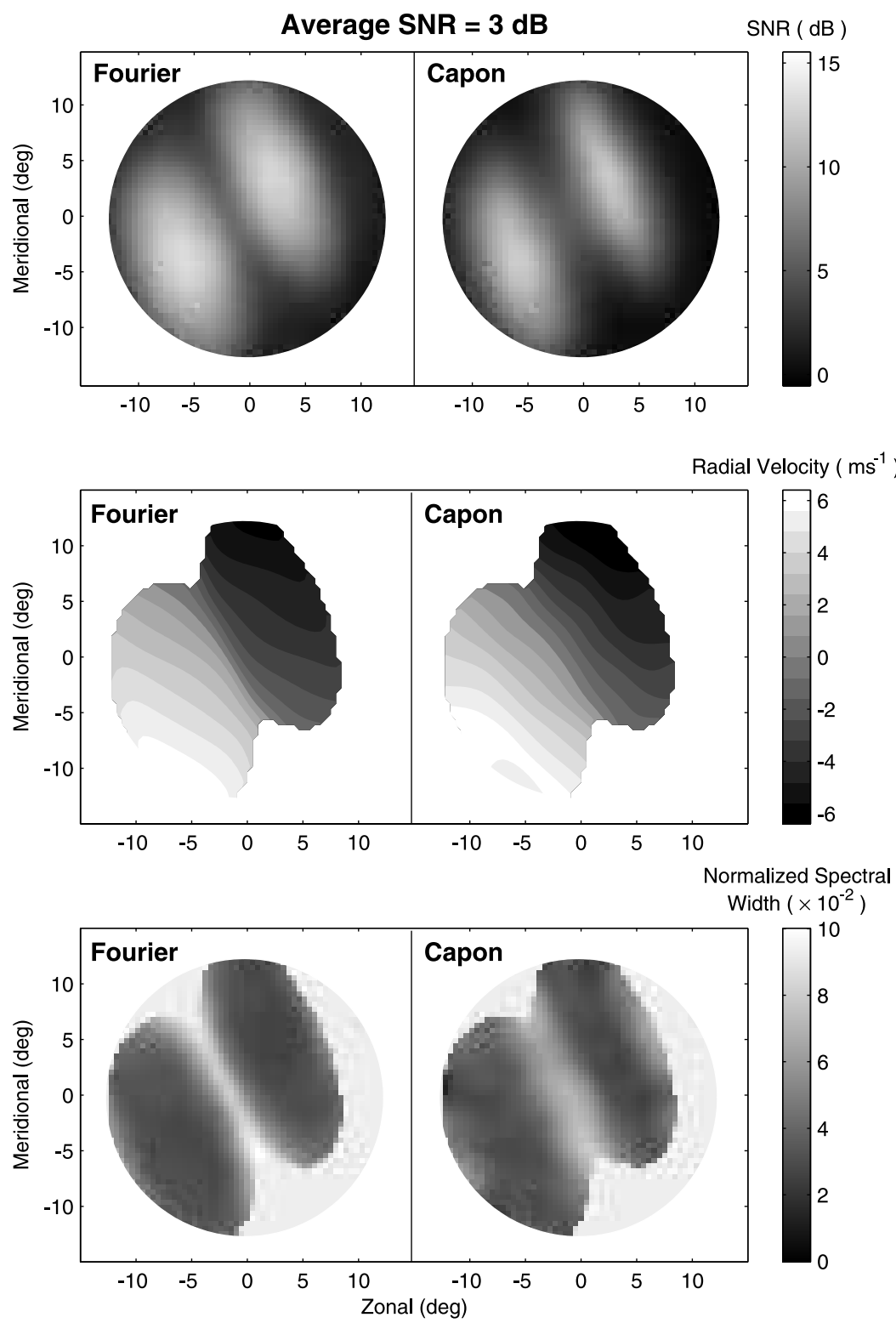

Figure 4. Echo power, radial velocity, and spectral width estimate using the PPB method. Radial velocity and spectral width maps are shown for the region with the SNR $>3 \mathrm{~dB}$.

scanned area, which is reasonable given the limited area covered by the nine beams.

[27] The well known minimum norm least squares solution for equation (11) is given by

$$
\mathbf{u}=\left(\mathbf{C}^{\dagger} \mathbf{C}\right)^{-1} \mathbf{C}^{\dagger} \mathbf{v}_{r}
$$

[28] Results obtained using equation (12) for estimates of the three-dimensional wind field are shown in
Figure 6. These results are generated for a situation in which there is a constant horizontal wind of $25 \mathrm{~ms}^{-1}$, no vertical velocity, and a turbulent RMS velocity of $\pm 1 \mathrm{~ms}^{-1}$ is added to the mean velocity. The corresponding wind field maps are obtained by computing the least square solutions for the nine linear equations in the $3 \times 3$ sliding window. A window distance of 3 pixels (from one middle pixel to the middle pixel in the adjacent $3 \times 3$ window) is used in this map. In a general sense, the estimates do give a reasonable indication of 


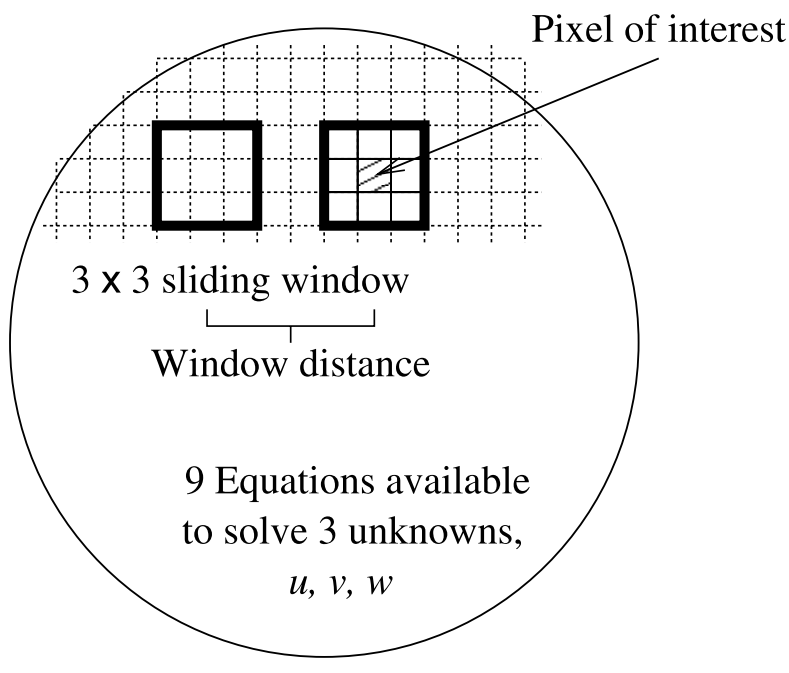

Figure 5. Wind field map is obtained by solving 9 linear equations for every pixel using a sliding window of $3 \times 3$ pixels. In this figure the window distance shown is 5 pixels. A minimum norm solution is obtained for this set of 9 linear equations.

the horizontal winds. The overall direction and approximate magnitudes are similar to the underlying known wind field. The actual horizontal wind vector is provided in the upper-right corner, for reference. Visually, the wind field estimates using the Fourier method seem to display a larger error than the Capon results. The RMS error for the Capon results $\left(5.427 \mathrm{~ms}^{-1}\right)$ appears to be significantly lower than that of the Fourier results $\left(8.131 \mathrm{~ms}^{-1}\right)$ at an SNR of $3 \mathrm{~dB}$. A set of experiments were run under the same conditions over a range of

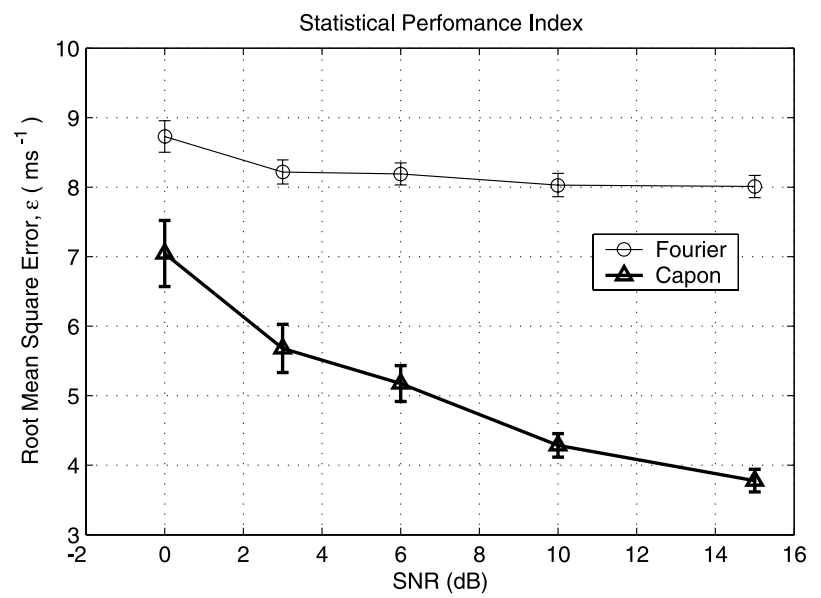

Figure 7. RMS errors of the two-dimensional horizontal wind field estimates from Fourier and Capon beamformers as a function of SNR at the receivers.

SNRs. These results are illustrated in Figure 7. Note the consistently lower RMS errors in the velocity estimates for the Capon technique. As SNR increases, the Fourier error does not change significantly, indicating that the source of the error is not predominantly noise.

[29] In Figure 8 the effect of angular variations in the underlying reflectivity pattern along the horizontal wind direction on the estimated radial velocity is illustrated. Assuming that the desired pointing direction is given by $\theta_{o}$, the observed signal extends over the range in which the receive beam pattern magnitude is significant. An ideal delta function beam pattern would sample the received signal from only the direction $\theta_{o}$ and yield a good estimate of the radial velocity in that direction. A

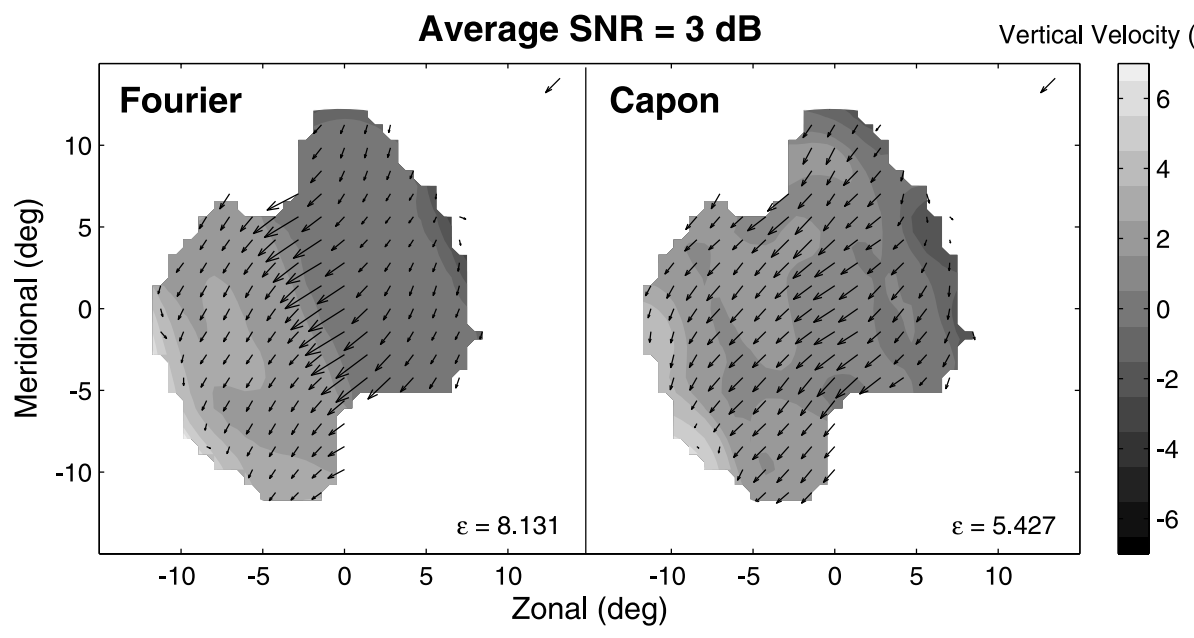

Figure 6. Three-dimensional wind field estimates obtained by computing the least squares solution for the nine radial velocity estimates from the new PPB method. 


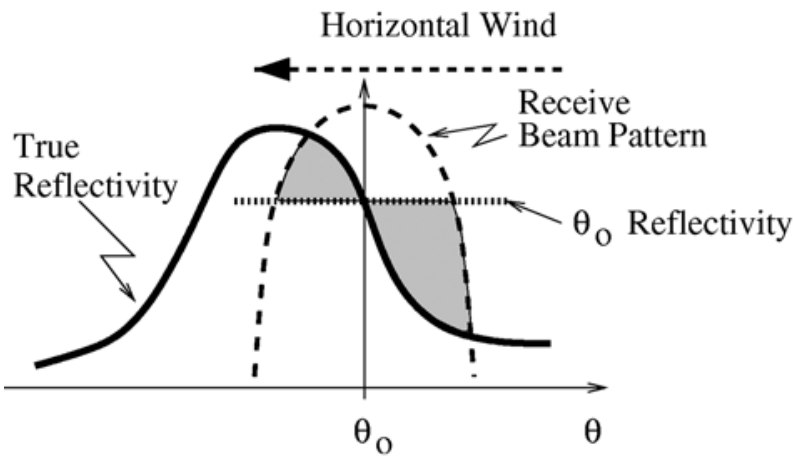

Figure 8. Graphical illustration of the effect of angular variations of the reflectivity with respect to horizontal wind. The shaded regions indicate the over and under weighting of radial velocities to the left and right of $\theta_{o}$, respectively. This weighting depends on the reflectivity distribution, the wind direction, and the antenna beam pattern and contributes a deterministic bias to the radial velocity estimates.

finite width beam will observe radial velocities over a range of angles. The variation of reflectivity along the direction of the horizontal wind will result in uneven weighting of velocities that are too low on one side of $\theta_{o}$ and too high on the other side of $\theta_{o}$. The shaded regions in the figure illustrate the extent to which the reflectivity variation contributes to a systematic bias in radial velocities.

[30] We can investigate the effects of reflectivity changes along the direction of the horizontal wind using
Fourier beamforming. Following a development similar to that used in May et al. [1988] for thin scattering layers, the biased estimated radial velocity can be written as

$\hat{v}_{r, \text { bias }}=\frac{\iint T(\theta, \phi) R\left(r_{o}, \theta, \phi\right) F(\theta, \phi)\left(\overrightarrow{\mathbf{v}}_{\mathrm{H}} \cdot \overrightarrow{\mathbf{u}}(\theta, \phi)\right) r_{o}^{2} \sin (\theta) \partial \theta \partial \phi}{\iint T(\theta, \phi) R\left(r_{o}, \theta, \phi\right) F(\theta, \phi) r_{o}^{2} \sin (\theta) \partial \theta \partial \phi}$

where $T(\theta, \phi)$ is the transmit beam magnitude, $R\left(r_{o}, \theta, \phi\right)$ is the true atmospheric reflectivity magnitude at range $r_{o}, F(\theta, \phi)$ is the Fourier based receive beam magnitude, $\overrightarrow{\mathbf{v}}_{\mathrm{H}}$ is the true horizontal wind velocity, and $\overrightarrow{\mathbf{u}}(\theta, \phi)$ is the unit direction vector toward direction $(\theta, \phi)$. The integration is taken over the small range of angles in which the receive beam is significant (i.e., the mainlobe region). The estimate $\hat{v}_{r \text {,bias }}$ can be used to generate an approximation to the error radial velocity estimate observed with the Fourier based simulation. These estimates are, to a first order approximation, equivalent to the errors observed in several different cases.

[31] Given the rather controlled nature of the conditions used in the experiment (constant, known horizontal velocity with weak turbulence), it is interesting to look at the horizontal wind estimates superimposed over the estimated reflectivity. Figure 9 presents these horizontal wind field estimates over the reflectivity estimates for both the Fourier and Capon based estimators. An interesting feature is observed near the valley between the two reflectivity peaks. Note the systematic over and under estimation compared to the known actual horizontal velocity indicated by the single arrow in the upper right

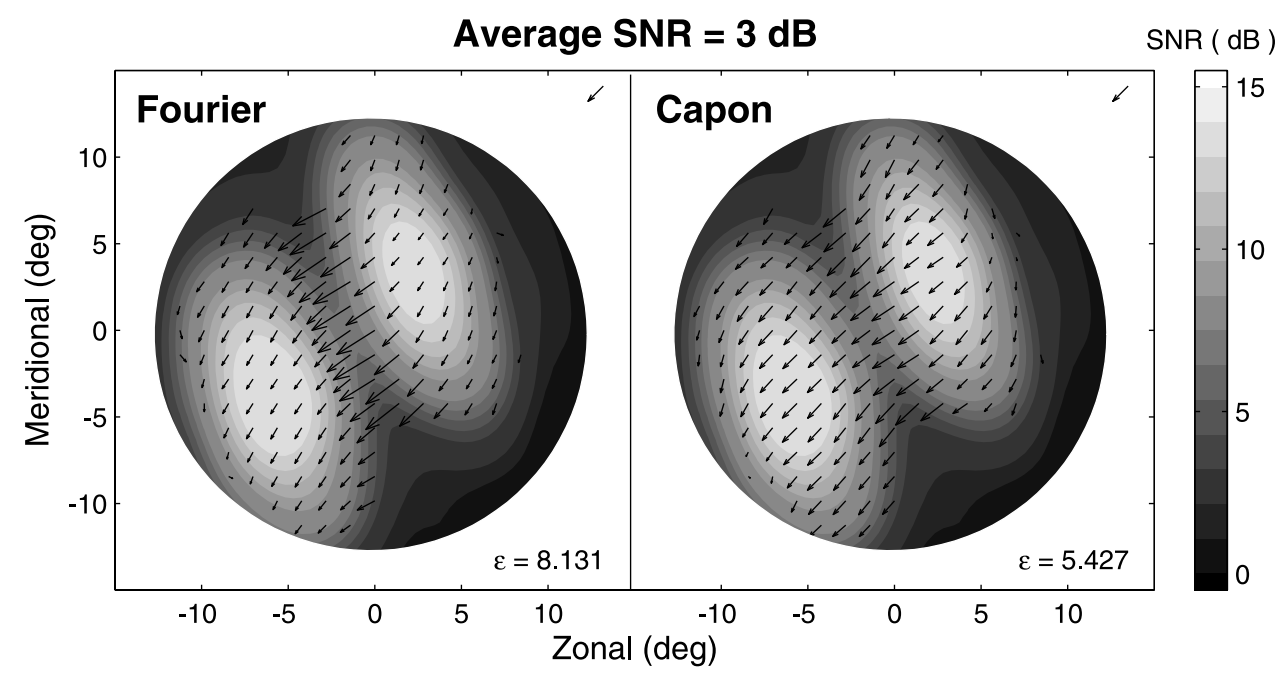

Figure 9. Two-dimensional horizontal wind field estimates superimposed over estimated reflectivity. Wind field estimates are obtained by computing the least squares solution for the nine radial velocity estimates from the new PPB method. 


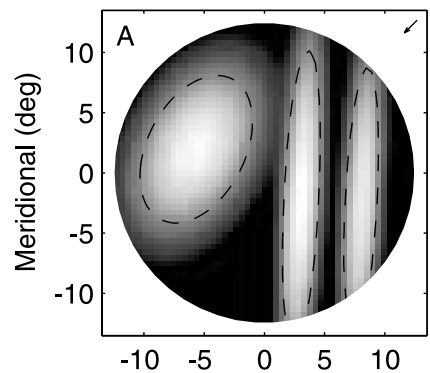

\section{Test Reflectivity Patterns}
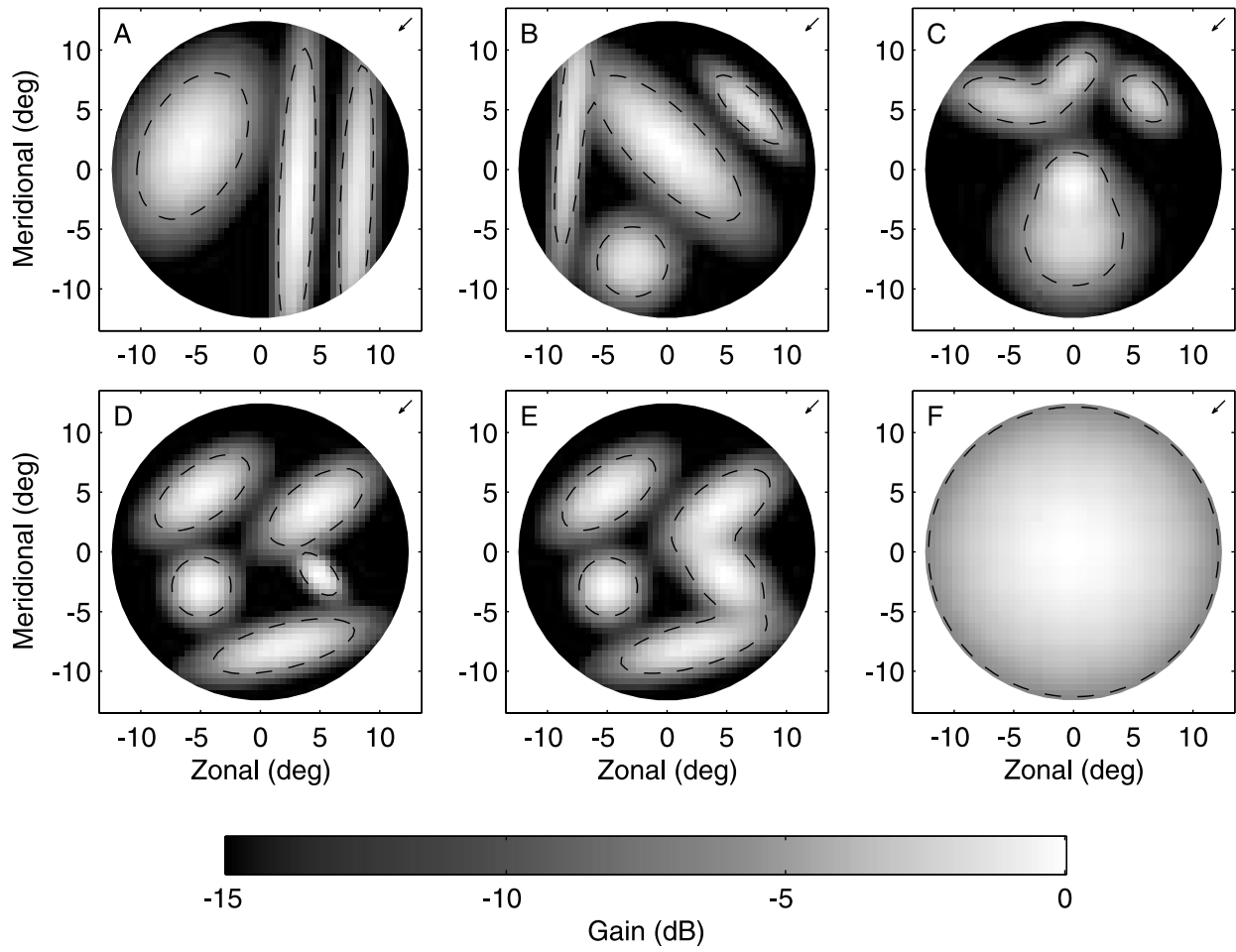

Figure 10. Six different reflectivity patterns were simulated to study the statistical performance of a variety of reflectivity fields. A uniform wind of $25 \mathrm{~ms}^{-1}$ with $\phi=45^{\circ}$ was used for all six experiments. The arrow in the upper right corner shows the true wind field vector. Patterns A through $\mathrm{E}$ were used to simulate random reflectivity field variations. In contrast, pattern $\mathrm{F}$ was used to simulate a homogeneous field for comparison. The wind fields obtained using Fourier PPB and Capon PPB are shown in Figure 11, and the RMS error velocities on these patterns are shown in Figure 12.

corner of each frame. Variations in the reflectivity pattern along the direction of the wind are accompanied by either systematic increases, decreases, or rotations in horizontal wind vector estimates. It appears that the variation is more significant in the case of the Fourier estimators than it is for the Capon estimators. Such errors have been known from some time in standard Doppler measurements [May et al., 1988]. Through the use adaptive array processing (Capon), however, it is now possible to mitigate these adverse effects for imaging applications.

\subsection{Quantitative Wind Field Results for Reflectivity Variations}

[32] The reflectivity pattern used in Figure 3 suggests the impact on the wind field estimates produced by reflectivity patterns. To investigate this possibility, another set of experiments with six different reflectivity fields were tested to assess the statistical performance of different reflectivity patterns. Figure 10 shows the six different reflectivity patterns that were used in this experiment. A uniform horizontal wind of $25 \mathrm{~ms}^{-1}$ with $45^{\circ}$ azimuth angle was used for all six patterns. Turbulent field of $0.3 \mathrm{~ms}^{-1}$ RMS velocity was added. Figure 11 shows the wind field estimates superimposed on the power distribution maps obtained from Fourier and Capon PPB methods. Note the poorer wind estimates from the Fourier PPB method especially around the regions with significant reflectivity variations. The images obtained from Capon PPB method do not show such poor results over much of the field. By varying the

Figure 11. Images of wind fields (superimposed on reflectivity maps) obtained using Fourier and Capon based PPB. The wind field estimates from Fourier based PPB algorithm suffers significantly from errors introduced by reflectivity field variations. Capon based PPB appears to mitigate these errors. 


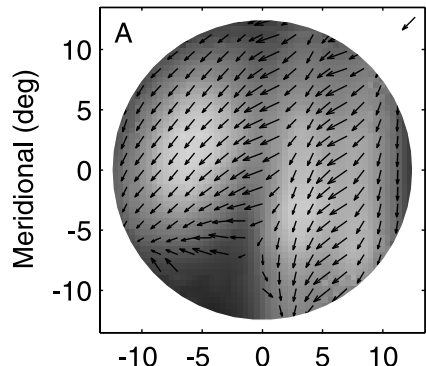

\section{Images from Fourier PPB}
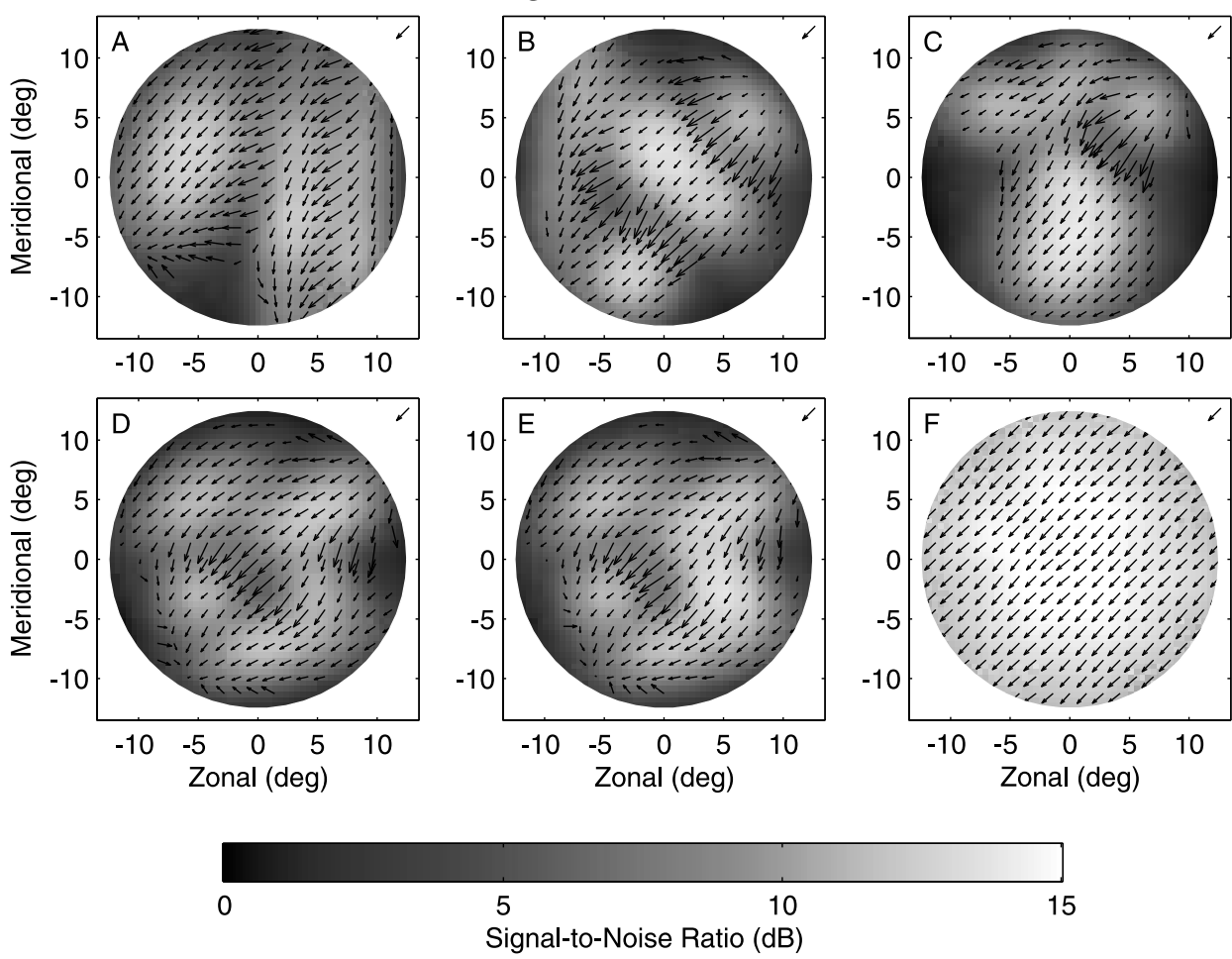

\section{Images from Capon PPB}
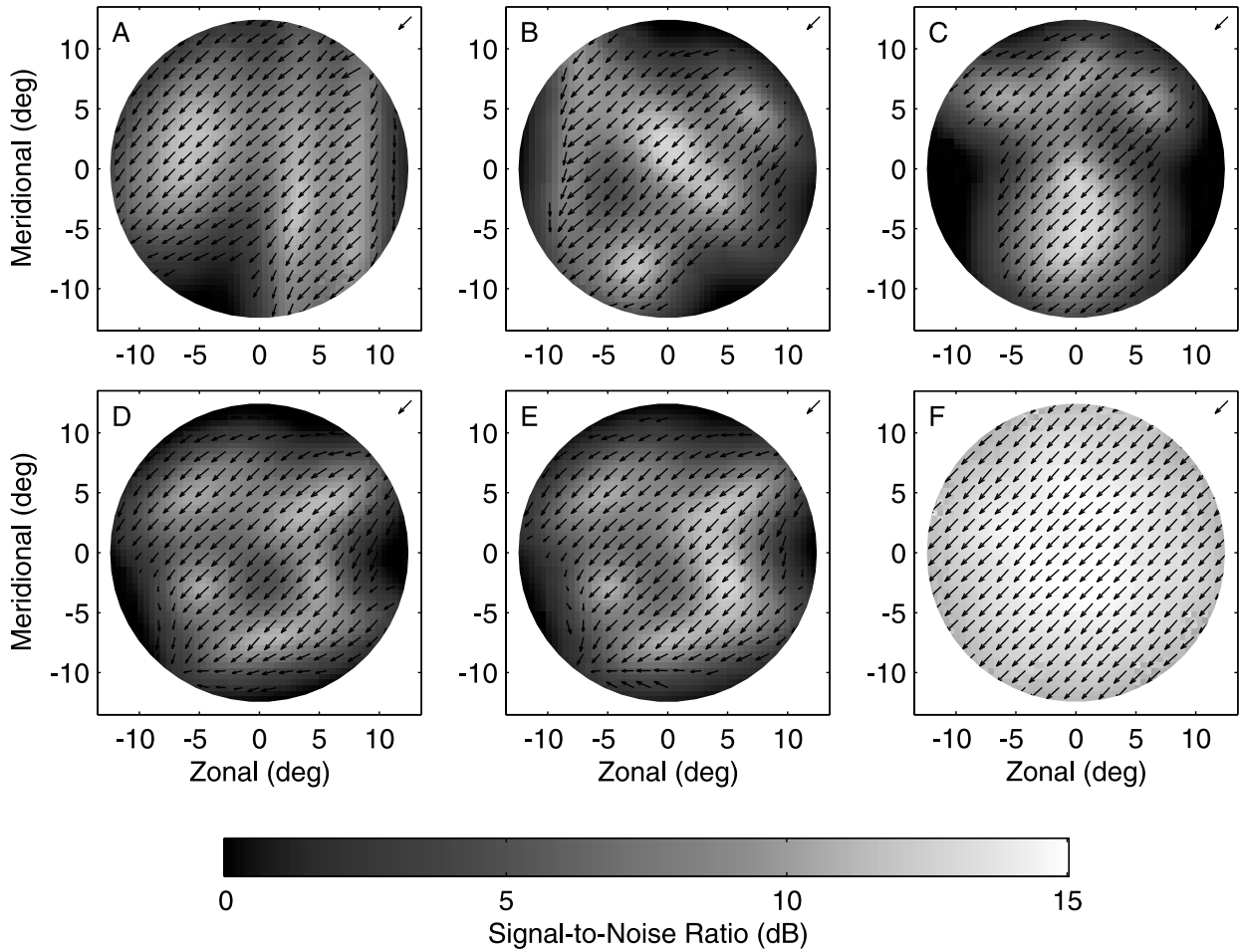

Figure 11

11 of 13 


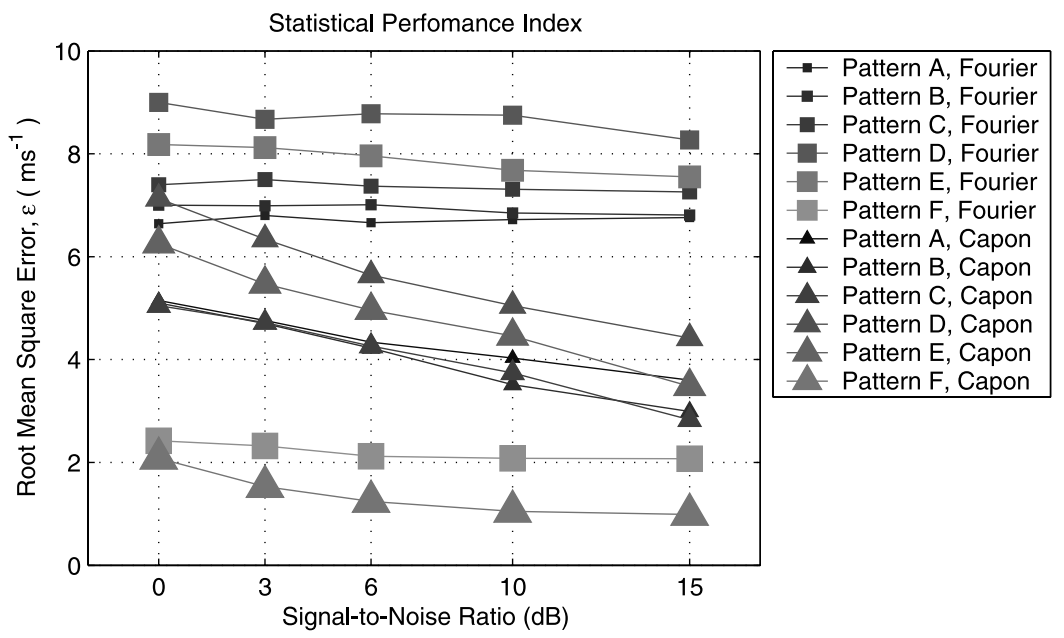

Figure 12. Fourier (squares) and Capon (triangles) RMS errors for the six test patterns in Figure 10 as a function of SNR.

SNR from 0 to $15 \mathrm{~dB}$, a statistical test was conducted for these six patterns. Figure 12 shows the RMS error of the wind field estimates for these six patterns as SNR is varied. Results from the Fourier PPB method do not improve with SNR but results from the Capon PPB method are initially better and continue to improve with increasing SNR. This motivates the use of Capon PPB method for imaging applications that require wind field maps as well as simply reflectivity maps.

\section{Conclusions}

[33] The primary contributions of this paper include a combination of pulse pair processing and beamforming techniques referred to as pulse pair beamforming (PPB) for estimating velocity and spectral width using beamforming techniques and a statistical investigation into the applicability of Capon and Fourier PPB techniques for high-resolution estimation of the wind and reflectivity field maps. The concept of pulse pair processing has been extended to beamformed imaging systems by using reflectivity derived weight vectors to estimate the pulse pair parameters necessary for both velocity and spectral width estimates. This PPB approach has been applied with both Fourier and Capon techniques. The validity of the PPB technique has been demonstrated by comparison to standard Doppler spectral techniques.

[34] While the Capon PPB technique has been shown to be superior in estimating velocities using simulated data, the varying nature of the adaptive beamformer beam pattern results in poor performance for spectral width applications. The fixed pattern Fourier technique appeared to be superior for the spectral width application. In addition, Capon PPB tends to consistently outperform Fourier PPB in statistical tests for three- dimensional wind field maps. A significant source of the errors that are mitigated by Capon PPB are reflectivity field variation induced errors in the wind field maps. These are visually apparent when the Fourier wind field maps are superimposed on the reflectivity maps. In addition, the constancy of the Fourier errors with increasing SNR are consistent with a signal induced (as opposed to noise induced) effect. Similar effects have been observed previously for the case of standard Doppler wind measurements [May et al., 1988].

[35] As pointed out earlier, existing MST radar systems and newly developed systems are beginning to routinely use CRI as a means of high-resolution studies of the atmosphere [Luce et al., 2001]. Even commercial systems are available with multiple receiver capabilities allowing CRI implementation. Given this interest in imaging technologies, statistical studies using realistic numerical simulations are becoming important. In fact, it is now apparent that the future of MST radar studies of the atmosphere will include developments in imaging techniques.

[36] Acknowledgments. B.L.C., R.D.P., and M.W.H. were supported by the Army Research Office through grant DDAD19-01-1-0407. S.J.F. and F.J.L.-D. were supported by the Army Research Office (Atmospheric Sciences) through grant DAAG55-98-1-0480.

\section{References}

Capon, J. (1969), High-resolution frequency-wavenumber spectrum analysis, Proc. IEEE, 57(8), 1408-1419.

Chau, J. L., and R. F. Woodman (2001), Three-dimensional coherent radar imaging at Jicamarca: Comparison of different inversion techniques, J. Atmos. Sol. Terr. Phys., 63, $253-261$. 
Cheong, B. L., M. W. Hoffman, and R. D. Palmer (2004), Efficient atmospheric simulation for high resolution radar imaging applications, J. Atmos. Oceanic Technol., 21, $374-378$.

Doviak, R. J., and D. S. Zrnić (1993), Doppler Radar and Weather Observations, Academic, San Diego, Calif.

Fukao, S., T. Sato, T. Tsuda, S. Kato, K. Wakasugi, and T. Makihira (1985a), The MU radar with an active phased array system: 1. Antenna and power amplifiers, Radio Sci., 20, 1155-1168.

Fukao, S., T. Tsuda, T. Sato, S. Kato, K. Wakasugi, and T. Makihira (1985b), The MU radar with an active phased array system: 2. In-house equipment, Radio Sci., 20, 11691176.

Haykin, S. (1985), Array Signal Processing, Prentice-Hall, Old Tappan, N. J.

Hélal, D., M. Crochet, H. Luce, and E. Spano (2001), Radar imaging and high-resolution array processing applied to a classical VHF-ST profiler, J. Atmos. Sol. Terr. Phys., 63, $263-274$.

Holdsworth, D. A., and I. M. Reid (1995), A simple model of atmospheric radar backscatter: Description and application to the full correlation analysis of spaced antenna data, Radio Sci., 30, 1263-1280.

Hysell, D. L. (1996), Radar imaging of equatorial $F$ region irregularities with maximum entropy interferometry, Radio Sci., 31(6), 1567-1578.

Hysell, D. L., and R. F. Woodman (1997), Imaging coherent backscatter radar observations of topside equatorial spread $F$, Radio Sci., 32, 2309-2320.

Hysell, D. L., M. Yamamoto, and S. Fukao (2002), Imaging radar observations and theory of type I and type II quasiperiodic echoes, J. Geophys. Res., 107(A11), 1360, doi:10.1029/ 2002JA009292.

Jain, A. R., Y. J. Rao, P. B. Rao, V. K. Anandan, S. H. Damle, P. Balamuralidhar, A. Kulkarni, and G. Viswanathan (1995), Indian MST radar: 2. First scientific results in ST mode, Radio Sci., 30, 1139.

Kudeki, E., and F. Sürücü (1991), Radar interferometric imaging of field-aligned plasma irregularities in the equatorial electrojet, Geophys. Res. Lett., 18(1), 41-44.

Kudeki, E., and R. Woodman (1990), A poststatistics steering technique for MST radar applications, Radio Sci., 25, 591594.

Luce, H., M. Crochet, and F. Dalaudier (2001), Temperature sheets and aspect sensitive radar echoes, Ann. Geophys., 19, 899-920.

May, P. T., S. Fukao, T. Tsuda, T. Sato, and S. Kato (1988), The effect of thin scattering layers on the determination of wind by Doppler radars, Radio Sci., 23, 83-94.

Mead, J. B., G. Hopcraft, S. J. Frasier, B. D. Pollard, C. D. Cherry, D. H. Schaubert, and R. E. McIntosh (1998), A volume-imaging radar wind profiler for atmospheric boundary layer turbulence studies, J. Atmos. Oceanic Technol., 15, 849-859.
Muschinski, A., P. P. Sullivan, D. B. Wuertz, R. J. Hill, S. A. Cohn, D. H. Lenschow, and R. J. Doviak (1999), First synthesis of wind-profiler signals on the basis of large-eddy simulation data, Radio Sci., 34(6), 1437-1459.

Palmer, R. D., M. F. Larsen, E. L. Sheppard, S. Fukao, M. Yamamoto, T. Tsuda, and S. Kato (1993), Poststatistic steering wind estimation in the troposphere and lower stratosphere, Radio Sci., 28, 261-271.

Palmer, R. D., K. Y. Lei, S. Fukao, M. Yamamoto, and T. Nakamura (1995), Weighted imaging Doppler interferometry, Radio Sci., 30, 1787-1801.

Palmer, R. D., S. Gopalam, T. Yu, and S. Fukao (1998), Coherent radar imaging using Capon's method, Radio Sci., 33, $1585-1598$.

Pfister, W. (1971), The wave-like nature of inhomogeneities in the $E$ region, J. Atmos. Terr. Phys., 33, 999-1025.

Pollard, B. D., S. Khanna, S. J. Frasier, J. C. Wyngaard, D. W. Thomson, and R. E. McIntosh (2000), Local structure of the convective boundary layer from a volume-imaging radar, J. Atmos. Sci., 57, 2281-2296.

Rao, P. B., A. R. Jain, P. Kishore, P. Balamuralidhar, S. H. Damle, and G. Viswanathan (1995), Indian MST radar: 1. System description and sample vector wind measurements in ST mode, Radio Sci., 30, 1125.

Röttger, J., and H. Ierkic (1985), Postset beam steering and interferometer applications of VHF radars to study winds, waves, and turbulence in the lower and middle atmosphere, Radio Sci., 20, 1461-1480.

Röttger, J., and R. A. Vincent (1978), VHF radar studies of tropospheric velocities and irregularities using spaced antenna techniques, Geophys. Res. Lett., 5, 917-920.

Woodman, R. F. (1971), Inclination of the geomagnetic field measured by an incoherent scatter technique, J. Geophys. Res., 76, 178-184.

Woodman, R. F. (1997), Coherent radar imaging: Signal processing and statistical properties, Radio Sci., 32(6), 23732391.

Yu, T.-Y. (2000), Radar studies of the atmosphere using spatial and frequency diversity, Ph.D. thesis, Univ. of Nebr., Lincoln.

Yu, T.-Y., R. D. Palmer, and D. L. Hysell (2000), A simulation study of coherent radar imaging, Radio Sci., 35(5), 11291141.

Yu, T.-Y., R. D. Palmer, and P. B. Chilson (2001), An investigation of scattering mechanisms and dynamics in PMSE using coherent radar imaging, J. Atmos. Sol. Terr. Phys., 63, 1797-1810.

B. L. Cheong, M. W. Hoffman, and R. D. Palmer, Department of Electrical Engineering, University of Nebraska, Lincoln, NE 68588-0511, USA. (boonleng@doppler.unl.edu)

S. J. Frasier and F. J. López-Dekker, Department of Electrical and Computer Engineering, University of Massachusetts, Amherst, MA 01003, USA. 